\title{
Developed Fingerprint Segmentation Technique based on Mean and Variance Intensity Thresholding
}

\author{
G.A. Bahgat ${ }^{\mathrm{a}}$, Alaa A. Hefnawy ${ }^{\mathrm{a}}$, A.H. Khalil ${ }^{\mathrm{b}}$, N.S. Abdel Kader ${ }^{\mathrm{b}}$, and S. Mashali ${ }^{\mathrm{a}}$ \\ ${ }^{a}$ Electronics Research Institute, Dokki, Giza, Egypt \\ ${ }^{\mathrm{b}}$ Faculty of Engineering, Cairo Univ., Giza, Egypt \\ *Alaa Hefnawy: alaa@eri.sci.eg
}

\begin{abstract}
Fingerprint segmentation is a preprocessing stage in the fingerprint recognition system. It is used to prevent the extraction of false features in the background or the low quality parts in the foreground (fingerprint area). Besides, it reduces the execution time of the recognition by considering only the region of interest. It is often preferred using simplified segmentation techniques to build the recognition systems. The most popular one is the variance based thresholding. This method has been developed in this paper through making it more robust while maintain the simplicity of design. It is proven to act more reliable in case of bad images.
\end{abstract}

\section{Introduction}

The fingerprint segmentation is the extraction of the fingerprint oriented lines from the background. The segmented part is called the foreground ${ }^{(1)}$. Fingerprint is produced by scanning the fingertip by an electronic sensor. The sensor types are capacitive, thermal and optical ${ }^{(2)}$. There can be areas inside the scanned fingerprint that do not contain oriented lines such as in the case of bruises that came from a skin disease or a burn in the skin or creases (small cuts). Also, the wet fingerprint can produce areas with connected ridges which will not show any lines as shown in Figure 1.b. These two kinds of area are unwanted for the feature extraction stage, thus, the segmentation method should segment these regions. A stage of fingerprint enhancement is present in some fingerprint recognition systems, which tries to predicates the shape of the ridges in these unwanted areas ${ }^{(3)}$. In the case of dry fingerprints, the ridge lines can be disconnected, as shown in Figure 1.c, it is assumed that an enhancement method used to connect these lines. The benefit of the segmentation stage is reduction in processing time of the fingerprint recognition processes for scanning the fingerprint area only ${ }^{(4)}$, and the prevention of extracting false features from the background ${ }^{(2)}$ or the unwanted regions as mentioned before. The segmentation should exclude the end points of the ridge lines at the boundary of the fingerprint to avoid the extraction of the false features.

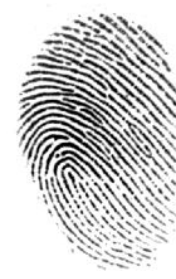

(a)

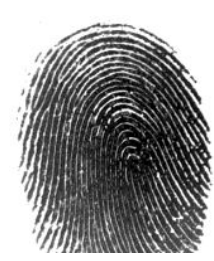

(b)

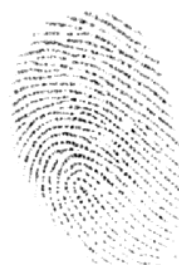

(c)

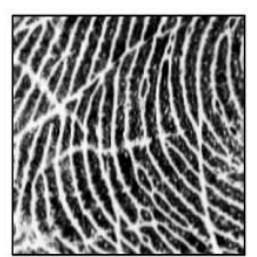

(d)
Figure 1: Fingerprint images: (a) good quality, (b) wet finger, (c) dry finger, (d) finger with creases.

There are several techniques that use the analysis of different features of the fingerprint in the segmentation process, such as the orientation coherence $e^{(1,5)}$, which is similar to the orientation consistency. Also, there are the local intensity variance ${ }^{(6)}$, the combination between the variance and the local intensity mean ${ }^{(7)}$, the combination between the coherence, local intensity mean and local intensity variance using a linear supervised classifier ${ }^{(8,9)}$ or $\mathrm{k}$-means clustering ${ }^{(10)}$, and the local intensity variance along and orthogonal to the ridge orientation ${ }^{(11-13)}$. Besides, there are the analysis of the local ridge orientation histogram ${ }^{(14)}$, the average magnitude of the local intensity gradient ${ }^{(15)}$, and the response of oriented Gabor filters ${ }^{(16)}$, which are based on the local ridge orientation and frequency. Since, a small fingerprint fragment resembles a two-dimensional sinusoid function, the analysis of its Fourier spectrum can also be used in segmentation ${ }^{(17,18)}$.

Recently, a combination of thresholding the local mean of the intensity and the local variance of the ridge gradient magnitudes applied in fingerprint segmentation ${ }^{(19)}$, followed by thresholding the gradient coherence to detect the noisy areas. Another method that depends on the image intensity directly, rather than the statistical features extracted from the image is presented in ${ }^{(20)}$. The method begins with applying a range filter which is a non-linear filtering method that replaces the original grey level of a pixel by the range value of the grey levels of the pixels in a specified neighborhood, followed by binarization method by using a Fuzzy C-means (FCM) clustering approach and 4 stage morphological post processing.

The fingerprint recognition systems mainly depends on simple segmentation technique; ${ }^{(21)}$ shows the improvement 
of using a convex Hull filter of the minutiae and the segmentation for the fingerprint in improving the state-ofthe-art mintuaie extractor, MINDTCT ${ }^{(22)}$ which is provided with NBIS software package, by 25\% EER (Equal Error Rate $)^{(2)}$ for the FVC databases. Atul ${ }^{(23)}$ perform fingerprint identification using three stage system: preprocess for enhancement, then thinning, then minutiae extraction using Crossing number concept. They use normalization followed by segmentation using variance thresholding. Runlin ${ }^{(24)}$ build embedded web authentication system using fingerprint recognition. it uses normalization followed by variance thresholding.

Our aim is to develop the simple variance threshold technique to be more robust and easily implemented on programmable systems such as FPGA.

\section{Simplified Segmentation and Morphological Operations}

\subsection{Simplified Segmentation Techniques}

The most common segmentation technique, used in the fingerprint recognition systems, is the intensity variance thresholding based technique ${ }^{(25-27)}$. It is characterized by its simplicity of design and effectiveness in fingerprint segmentation.

The procedure, given $\mathrm{in}^{(6)}$, starts with dividing the fingerprint image into non overlapping blocks (or windows). For each block, the local mean and variance of the intensity values are calculated. Then, the segmentation mask is formed of the blocks with local variance, greater than or equals a variance threshold. Finally, a smoothing technique is applied on the segmentation mask $S m$ to wipe off the isolated image blocks by the following equation:

$$
S m_{s}(i, j)=\frac{\sum_{k=i-1}^{k=i+1} \sum_{l=j-1}^{l=j+1} \operatorname{Sm}(k, l)}{n \times n}
$$

where $S m_{s}$ is the smoothed segmentation mask and $n$ is the size of the smoothing window.

Chen $^{(7)}$ uses the intensity mean information and segments the blocks according to a global mean limiter according to the following equation:

$\operatorname{Sm}(i, j)=\left\{\begin{array}{l}1 \quad \text { if } \quad \operatorname{var}_{l}(i, j) \leq v_{\text {th }} \quad \text { and } m_{g L} \leq \text { mean }_{l}(i, j) \leq m_{g H} \\ 0 \quad \text { otherwise }\end{array}\right.$

where, $m_{g L}$ and $m_{g H}$ are the global lower and upper mean thresholds, respectively. Besides, it applies morphological operations including dilation and erosion to remove some isolated points in the background and to fill large holes inside the foreground.

The segmentation procedure given $i^{(22)}$ combines the concepts presented $\mathrm{in}^{(3,8,28)}$. The fingerprint image is globally normalized $^{(3)}$, they say that this will allow setting a global threshold for all the images. The local variance is calculated after dividing the image into blocks with size
( $8 \times 8$ pixels). Then, global variance thresholding is applied to segment the fingerprint, which is a part from ${ }^{(8)}$ and work. Refinement using three iteration of hole filling and erosion is applied.

\subsection{Morphological Operations}

The morphological operations are used to improve the performance of the segmentation. They can remove isolated points and fill holes in the segmentation mask ${ }^{(7,29)}$.

Morphology is a set of image processing operations that process images based on shapes. In a morphological operation, the value of each pixel in the output image is based on a comparison of the corresponding pixel in the input image with its neighbors. Two operations are used: dilation is an operation that adds pixels to the boundaries of the objects in the image, and erosion is an operation that removes pixels on the object boundaries. The number of pixels added or removed from the objects depends on the size and shape of an element called the structuring element used to process the image. A structuring element is a matrix consisting of only zero and one values. It can have any arbitrary shape and size, where the pixels with values of one define the neighborhood. Structuring elements are much smaller than the image being processed. The center pixel of the structuring element, called the origin, identifies the pixel of interest (the pixel being processed). In Dilation, the value of the output pixel is the maximum value of all the pixels in the input pixel's neighborhood. In erosion, the value of the output pixel is the minimum value of all the pixels in the input pixel's neighborhood. An example of a segmented mask passing through the morphological opesrations is shown in Figure 2.

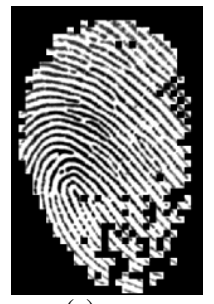

(a)

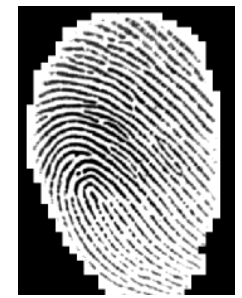

(b)

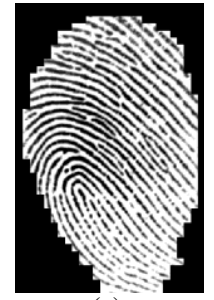

(c)
Figure 2: Morphological operations processed on segmented fingerprint: (a) segmented image, (b) image after dilation, (c) image after erosion

\section{Proposed Developed Segmentation}

\subsection{Analysis}

There are two problems that occurred for the variance thresholding techniques that are solved in the proposed work. First, the use of one global value or thresholding leads to the reduction of the segmentation performance in the dry fingerprints, where the ridges are of low contrast as shown in Figure 3. 


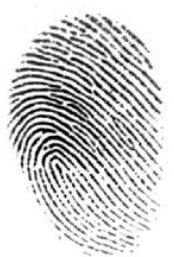

(a)

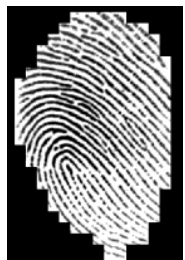

(b)

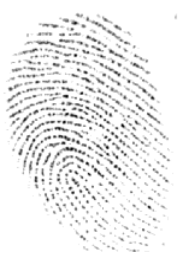

(c)

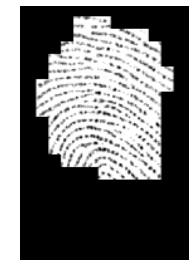

(d)
Figure 3: Effect of the use of one global value for thresholding: (a) fingerprint image, and (b) its segmented shape, (c) a dry condition, and (d) its segmented shape

Second, while adjusting the variance threshold $v_{t h}$ on the images in FVC2002 DB1 database, and testing it on DB2 database, it was found that there is a need of re-adjusting the variance thresholding as shown in Figure 4. The difference between the two databases is the sensor type

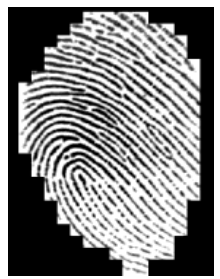

(a)

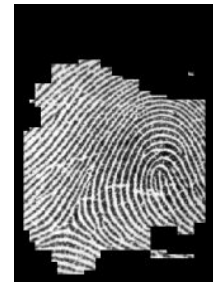

(b)
Figure 4: Effect of sensor on the fingerprint segmentation ( $v$ th =1500): (a) segmented fingerprint from DB1, (b) segmented fingerprint from DB2

The proposed solution is to define the local intensity variance with respect to the global variance using min-max normalization by a value $N_{\min \text {-max }}$, which is given by:

$$
N_{\text {min-max }}=\text { var }_{\text {max }}-\mathrm{var}_{\text {min }}
$$

where $v r_{\text {max }}$ and $v r_{\text {min }}$ are the maximum and minimum local variance values in an image, respectively. Thus, when the normal and dry impression, of Figure 3, is segmented using the normalized variance, the segmented images for the same vth are shown in Figure 5. But, there is still a problem at the fingerprint border. It is undesirable to have endpoints at the border because it will lead to the false detection of minutiae (end point type). Besides, reference ${ }^{(9)}$ subtracted the local mean by the global mean $m_{g}$, to solve the problem of dry and wet conditions of the fingerprint. It is found that this subtraction can be used to reduce the segmentation areas at the fingerprint boundary, by putting a condition on the local mean intensity.
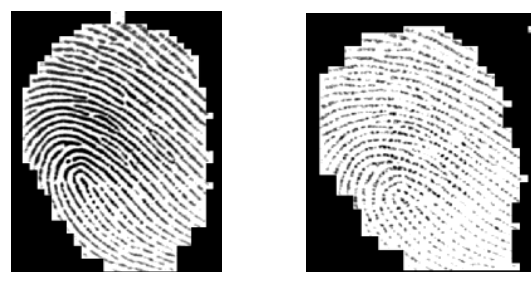

Figure 5: The effect of normalized variance on the dry fingerprint
The histograms of the intensity mean are analyzed to consider the importance of using the mean threshold. An example of the histogram of the local mean of the foreground and background of a sample of fingerprint images is shown in Figure 6. The background intensity values are usually high, thus, a condition can be put on the local mean $m_{g H}$, but no need for the lower mean threshold $m_{g L}$. The mean thresholding, denoted by $m_{t h}$ can be used to discriminate between the foreground and the background as shown in Figure 6. The intensity values are already scaled using the gray scale (0-255). The mean intensity histogram of the background of NIST4 database has two peaks; the peak at intensity value 255 represents the white space located at the bottom of the images as shown in Figure 7, and the second peak presents the background of the card of the inked fingerprint.

Finally, the structuring element size of the morphological operations; the dilation and erosion, are taken different. The dilation should close small holes in the segmentation mask by extending the mask boundary. Then, the erosion process should return the mask to its original boundary. But taking into consideration that, it is required to exclude the ridge end points from the fingerprint boundary, thus, the erosion element size strl is more than the dilation element size str2, such that:

$$
\text { strl }<\text { str } 2
$$

\subsection{Procedure}

Consequently, the proposed segmentation procedure is given by:

1. The fingerprint image global intensity mean $m_{g}$ is calculated by:

$$
m_{g}=\frac{1}{M \times N} \sum_{M, N} I(x, y)
$$

where $I(x, y)$ is the gray scale intensity of the fingerprint image at pixel $(x, y)$, and $M$ and $N$ are the size of the fingerprint image.

2. The fingerprint image is divided into non-overlapping blocks of size $w \times$ w pixels.

3. For each block, the local biased mean mean $n_{l b}$ of the block $(i, j)$ is calculated using (5) as:

$$
\operatorname{mean}_{l b}(i, j)=\frac{\sum_{x=i-b}^{x=i+b} \sum_{y=j-b}^{y=j+b} I(x, y)}{w \times w}-m_{g}
$$

4. The local intensity variance $v a r_{l}(i, j)$ is given by:

$$
\operatorname{var}_{l}(i, j)=\frac{\sum_{x=-b}^{b} \sum_{y=-b}^{b}\left(I(x, y)-\operatorname{mean}_{l b}(i, j)\right)^{2}}{w \times w}
$$

where $b=(w-1) / 2$.

5. The normalized local variance $\operatorname{var}_{n l}(i, j)$ is calculated using equations (8) and (7) as follows: 
$\operatorname{var}_{n l}(i, j)=\left\{\begin{array}{cl}\frac{\operatorname{var}_{l}(i, j)}{N_{\min -\max }} & \text { if } \text { mean }_{l}(i, j) \leq m_{t h} \\ 0 & \text { otherwise }\end{array}\right.$

6. The segmented mask $S$ is calculated as:

$$
S(i, j)=\left\{\begin{array}{lr}
1 & \operatorname{var}_{n l}(i, j) \leq v_{t h} \\
0 & \text { otherwise }
\end{array}\right.
$$

7. Morphological operations are used. A structuring element of square shape. Dilation process with structuring element length $s t r l$, and followed by erosion with structuring element length str2, are applied. Thus, obtaining the final segmented mask $S m$

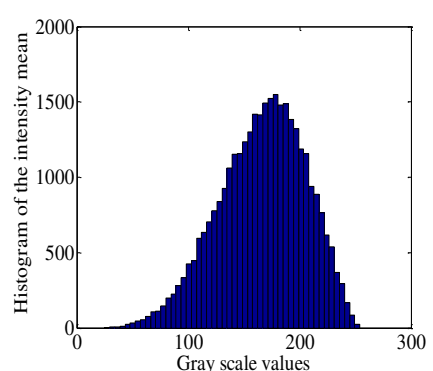

(a)
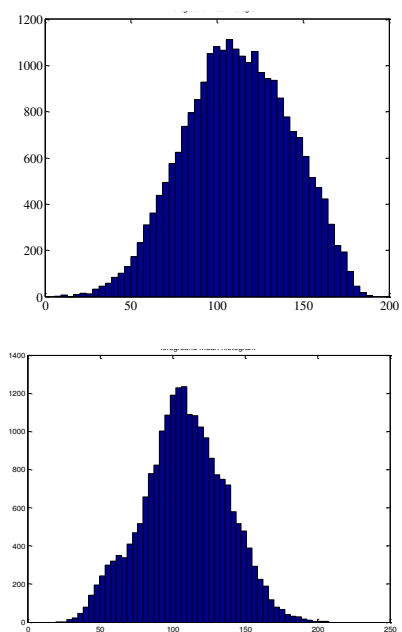

(e)

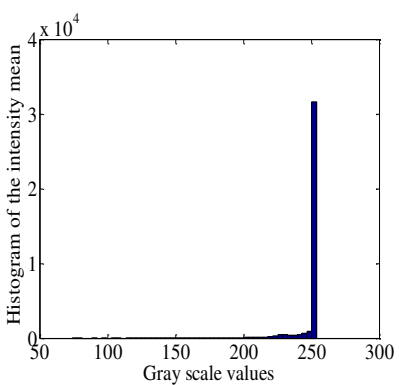

(b)
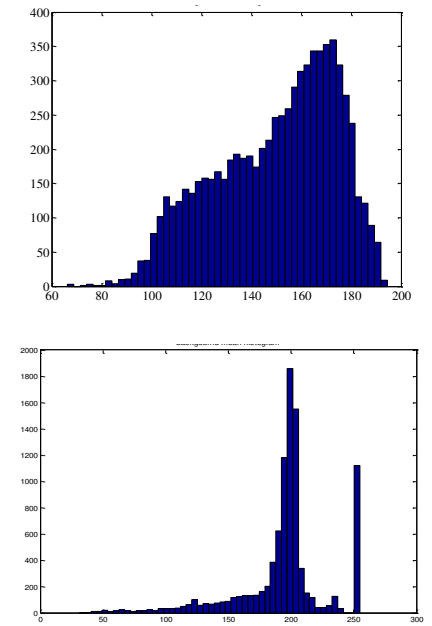

(f)
Figure 6: The mean histograms of FVC2002 DB1 (a) the foreground, and (b) the background, DB2 (c) the foreground, and (d) the background), and NIST4 (e) the foreground, and (f) the background

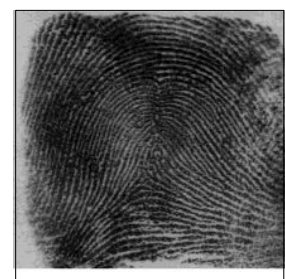

Figure 7: NIST4 fingerprint image contains white space at the bottom

\section{Experimental Results}

First, the block size $w$ is chosen to be an odd number to have a center pixel for the block. This size should be greater than the ridge width to obtain a variance value at the ridge/valley edge. Thus, the block size $w$ depends on the ridge width of the fingerprint, or more practically, the ridge to ridge period. Reference [27] uses (9) block size $w=8$ for the FVC database. In our work, it is chosen $w=9$, and according to equation (4), the structuring element size of the dilation process $s t r 1$ is taken as $3 \times 3$ blocks and that of the erosion process is taken $5 \times 5$ blocks.

\subsection{Manual Segmentation}

A manual segmentation of selected images from set (B) is done in FVC2002 DB1 and DB2 databases ${ }^{(30)}$ in order to use it in the parameter tuning of the unknown parameters: the mean threshold $\mathrm{m}_{\mathrm{th}}$ and the variance threshold $\mathrm{v}_{\mathrm{th}}$. References [10] and [31] use ten randomly selected fingerprints from each database. Bazen ${ }^{(8)}$ selected the first impression of thirty fingerprints. From our point of view, all impressions of the fingerprint should be considered in the training set, since these impressions are taken in different conditions. Thus, forty fingerprints (five fingers with their eight impressions) in FVC2002 DB1A and sixteen fingerprints from $\mathrm{FVC} 2002 \mathrm{DB} 2 \mathrm{~B}^{(30)}$, and the first ten (five fingers with their two impressions) in the inked NIST4 database $^{(32)}$ are segmented manually. This is done by drawing a line that segments the fingerprint area from the background and very low contrast areas. The background is colored with a distinctive color, for example, "red". Then, the manual segmented fingerprint is divided into blocks of size $w$; each block is labeled a background if at least one pixel in the block contains the distinctive color, otherwise, the block is labeled as foreground. Examples of the manual segmented fingerprints are shown in Figure 8.

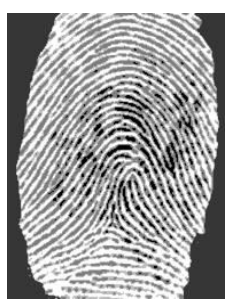

(a)

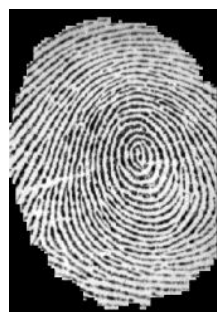

(c)

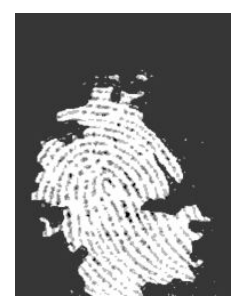

(b)

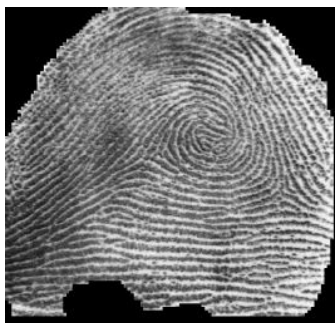

(d)
Figure 8: Manual segmented fingerprints: FVC2002 DB1

(a) good quality, (b) bad quality, (c) DB2, (d) NIST4 


\subsection{Parameter Tuning}

\subsubsection{Intensity Mean}

The mean intensity histograms of the fingerprint images, after the subtraction, are shown in Figure 9. The biased mean threshold is calculated by assuming the fingerprint segmentation will depend on the intensity mean only, such that by considering equation (6), the segmentation mask will be calculated by the given equation:

$$
S(i, j)=\left\{\begin{array}{cl}
1 & \text { if } \text { mean }_{l b}(i, j) \leq m \text { th } \\
0 & \text { otherwise }
\end{array}\right.
$$

The segmentation error $E_{s}$ is defined by the given equation ([Yang et al., 2012] and [Yang et al., 2010]):

$$
E_{s}=\frac{\text { number of missclassified blocks }}{\text { total number of blocks }} \times 100
$$

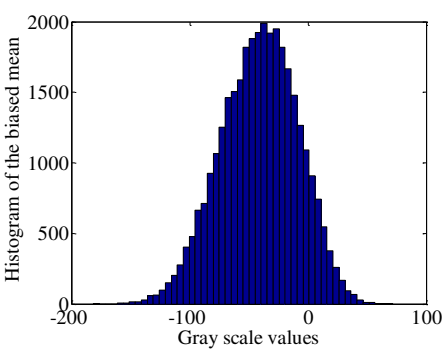

(a)

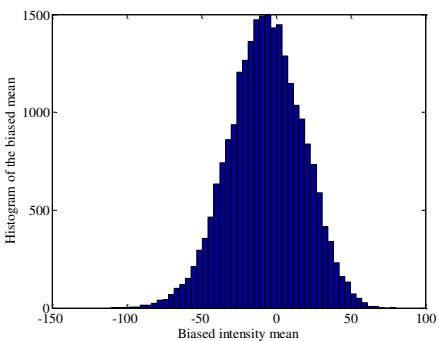

(c)

(e)

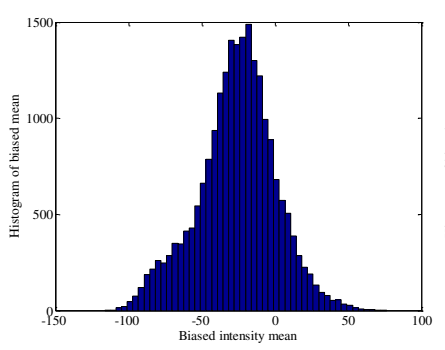

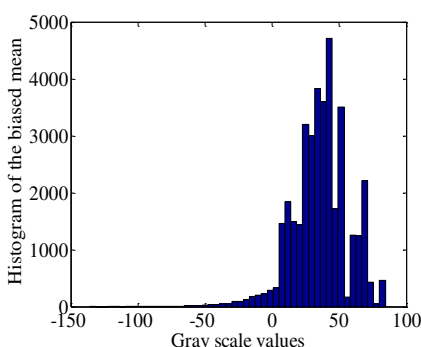

(b)

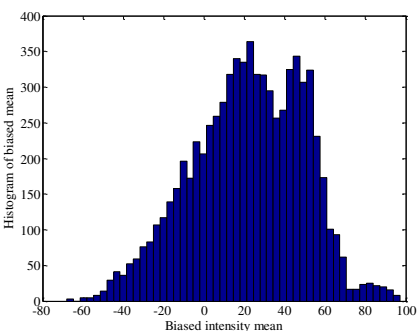

(d)

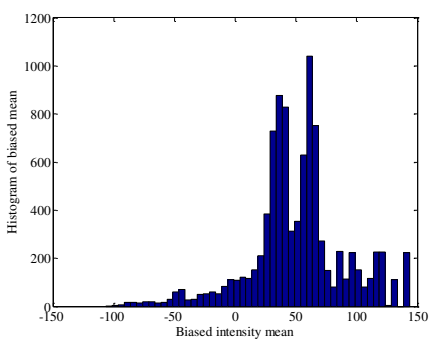

(f)
Figure 9: The biased mean histograms of FVC2002 DB1 (a) the foreground, and (b) the background, DB2 (c) the foreground, and (d) the background), and NIST4 (e) the foreground, and (f) the background.
The results are shown in the curves given in Figure 10. The optimum mean threshold that is equivalent to the minimum segmentation error for each database is given in Table 1, with the chosen mean threshold $(m t h=30)$ and the equivalent segmentation error. As, for the inked database, the mean threshold is not effective as shown in Figure 10. It is chosen $m t h=100$

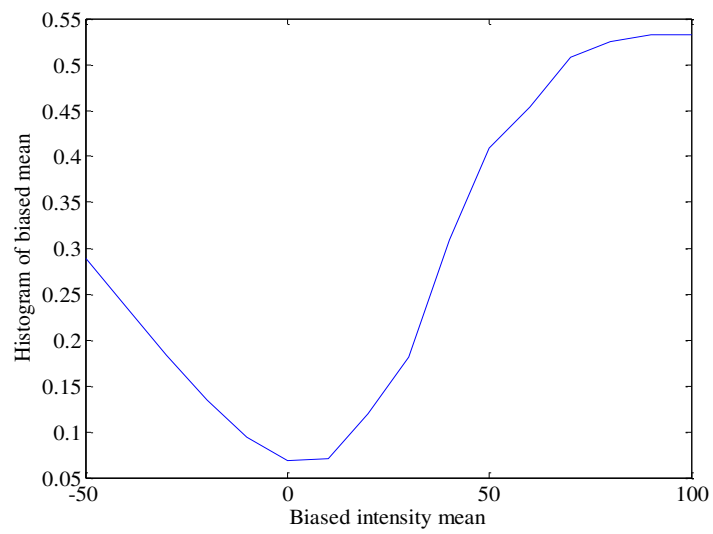

(a)

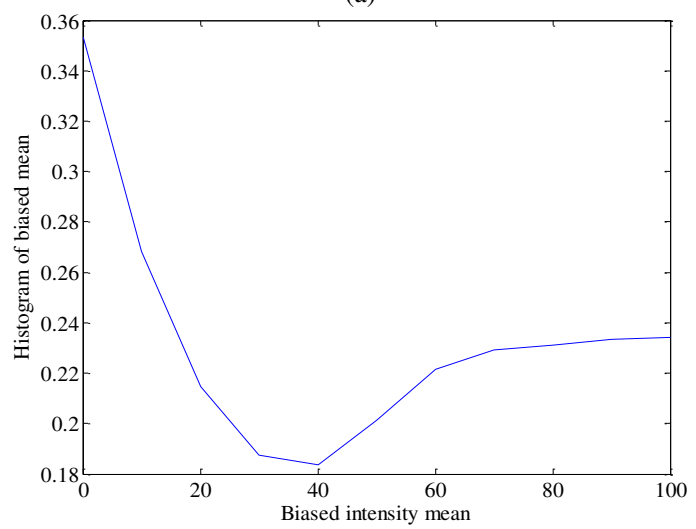

(b)

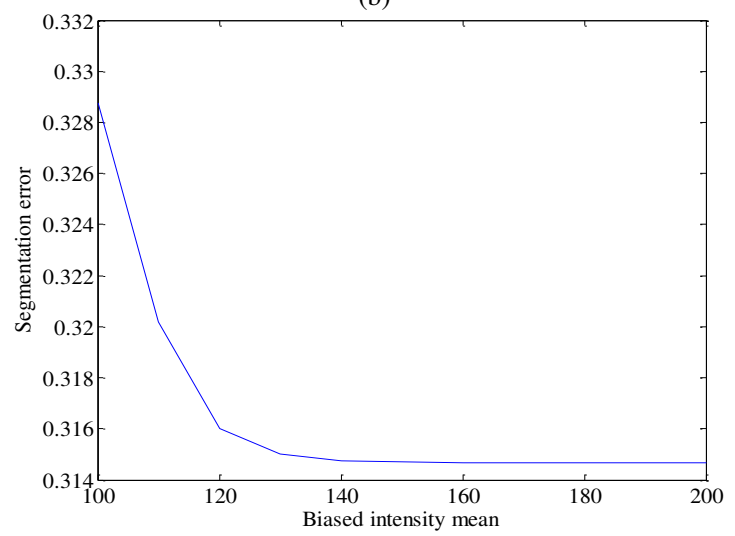

(c)

Figure 10: The segmentation error against the mean threshold values for a sample of (a) FVC2002DB1, (b) FVC2002 DB2, and (c) NIST4 databases 
Table 1: Biased intensity mean threshold values

\begin{tabular}{|l|l|l|l|l|}
\hline Database & $\begin{array}{l}\text { Optimum } \\
m t h\end{array}$ & $E_{s}$ & $\begin{array}{l}\text { Chosen } \\
m t h\end{array}$ & $E_{s}$ \\
\hline $\begin{array}{l}\text { FVC2002 } \\
\text { DB1 }\end{array}$ & 0 & 0.06818 & 30 & 0.181 \\
\hline $\begin{array}{l}\text { FVC2002 } \\
\text { DB2 }\end{array}$ & 40 & 0.1838 & 30 & 0.1877 \\
\hline NIST4 & 160 & 0.3147 & 100 & 0.3287 \\
\hline
\end{tabular}

\subsubsection{Intensity Variance}

The histogram of the intensity variance of the fingerprints is analyzed. An example of the histogram of the local variance of the foreground and background of a sample of fingerprint images is shown in Figure 11. The variance of the background is very low, while the variance of the fingerprint area is high. The normalized variance values are shown in Figure 12, which does not affect the histogram shape of the foreground and the background.

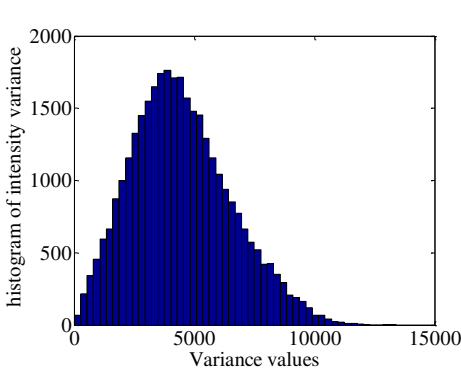

(a)

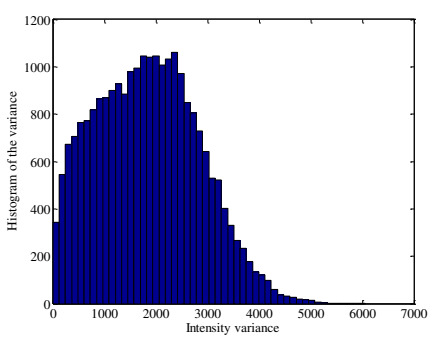

(c)

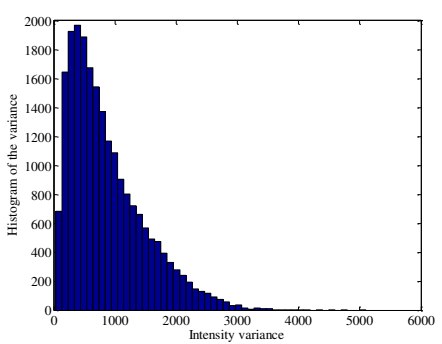

(e)

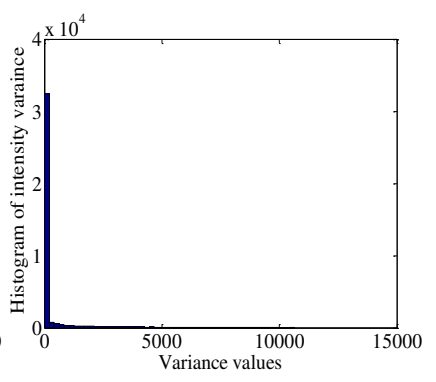

(b)

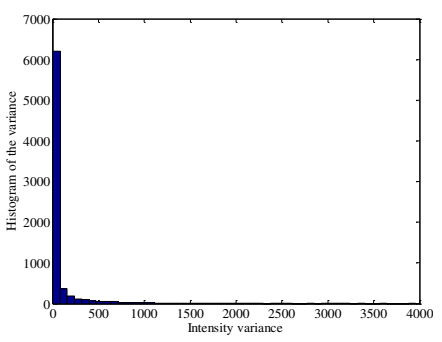

(d)

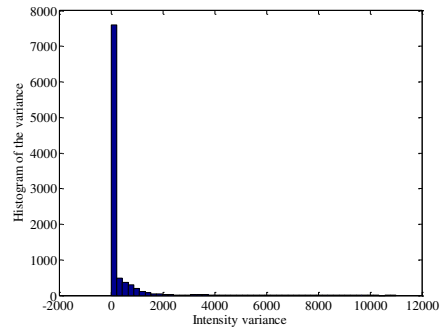

(f)
Figure 11: The histogram of the variance of FVC2002 DB1 (a) the foreground, and (b) the background, FVC2002 DB2

(c) the foreground, and (d) the background, and NIST4 (e) the foreground, and (f) the background

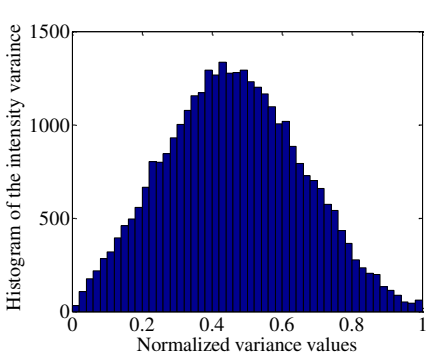

(a)

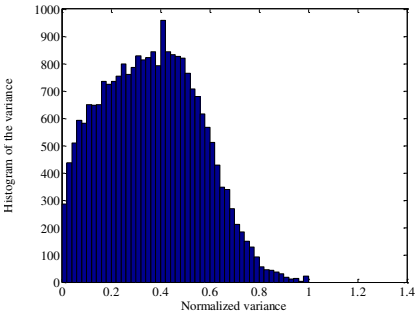

(c)

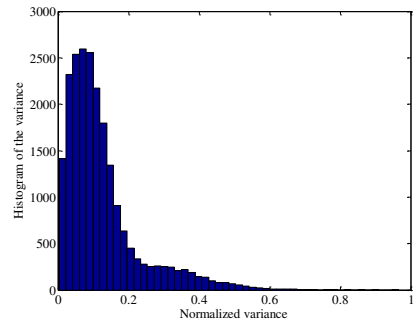

(e)

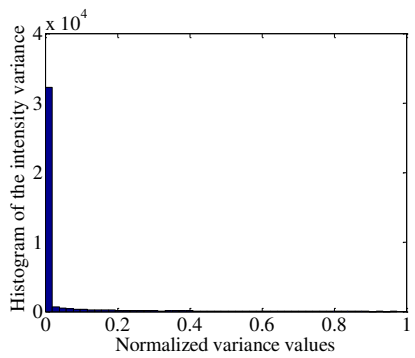

(b)

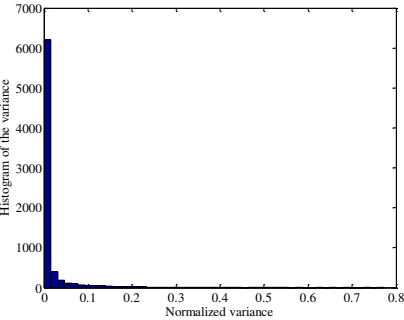

(d)

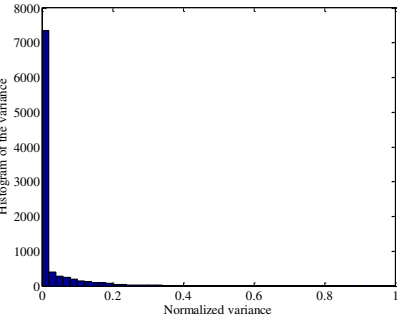

(f)
Figure 12: The histogram of the normalized variance of FVC2002 DB1 (a) the foreground, and (b) the background, FVC2002 DB2 (c) the foreground, and (d) the background, and NIST4 (e) the foreground, and (f) the background

The segmentation is performed using equations (8) to (9). The segmentation error $E_{s}$, defined by equation (11) is calculated for different values of the variance threshold. The results are shown in the curves given in Figure 13. The optimum variance threshold that is equivalent to the minimum segmentation error for each database is given in Table 2, with the chosen variance threshold $(v t h=0.02)$ and the equivalent segmentation error. The effectiveness of the mean thresholding for the inked fingerprints is shown in Figure 14, where the segmentation error for a specific variance threshold will be more in case of not applying the mean threshold. Thus, the mean thresholding is used in case of the inked images.

Table 2: Normalized intensity variance threshold values

\begin{tabular}{|l|l|l|l|l|}
\hline Database & $\begin{array}{l}\text { Optimum } \\
\text { vth }\end{array}$ & $E_{s}$ & $\begin{array}{l}\text { Chosen } \\
\text { vth }\end{array}$ & $E_{s}$ \\
\hline $\begin{array}{l}\text { FVC2002 } \\
\text { DB1 }\end{array}$ & 0.02 & 0.1055 & 0.02 & 0.1055 \\
\hline $\begin{array}{l}\text { FVC2002 } \\
\text { DB2 }\end{array}$ & 0.03 & 0.04168 & 0.02 & 0.04259 \\
\hline NIST4 & 0 & 0.08853 & 0.02 & 0.1781 \\
\hline
\end{tabular}




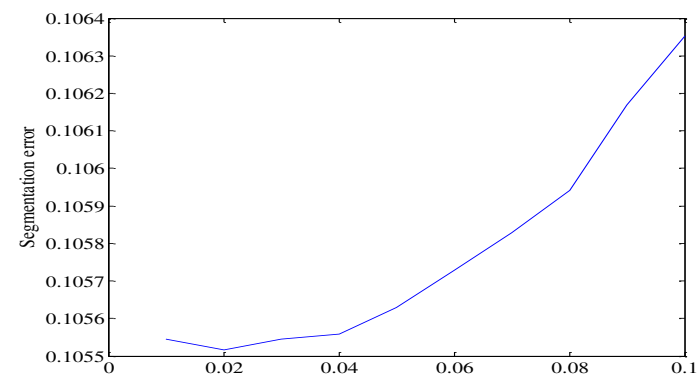

(a)

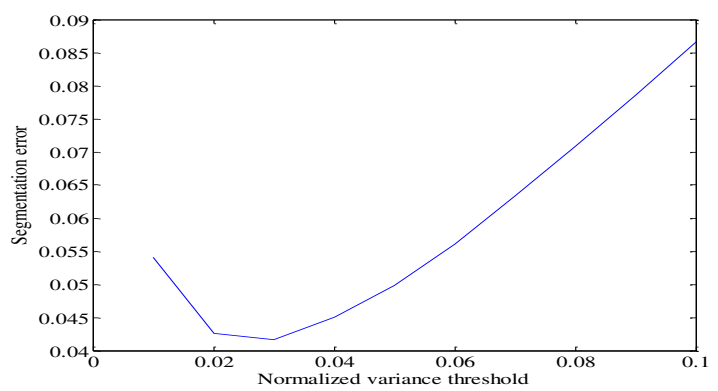

(b)

Figure 13: The segmentation error against the normalized variance threshold for a sample of (a) FVC2002DB1, (b) FVC2002 DB2, and (c) NIST4 databases

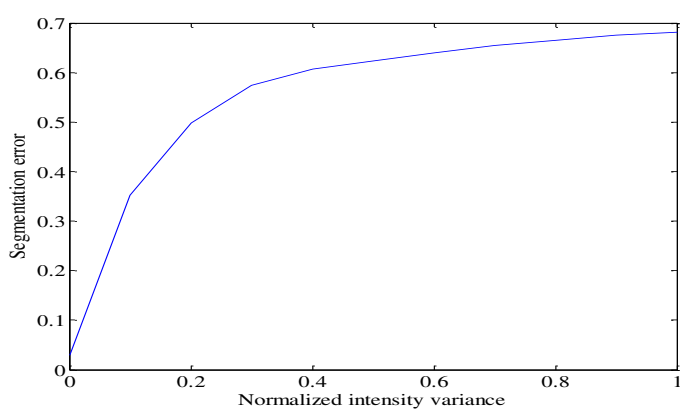

(a)

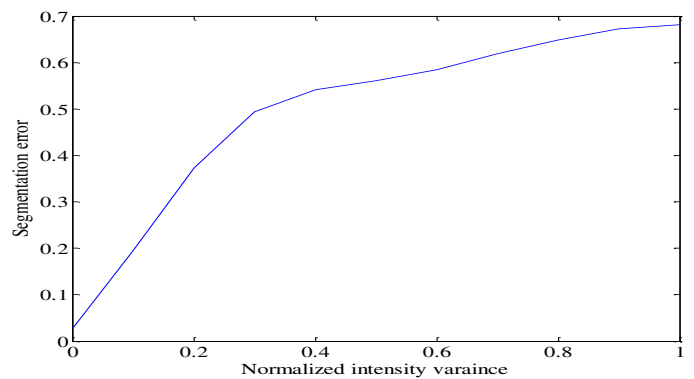

(b)

Figure 14: The segmentation error against the normalized variance threshold for a sample of NIST4 images: (a) without mean thresholding, and (b) with mean thresholding $(m t h=100)$

\subsubsection{Results}

A different database is used to evaluate the segmentation technique: the manual masks, given in the FOE (Fingerprint Orientation Extraction) benchmark ${ }^{(33)}$, are used to test the accuracy of the modified segmentation method. The parameter values are $w=9, s t r l=3, s t r 2=5, \quad m t h=30$, and $v t h=0.02$. The average computation time and the average error of the segmentation techniques of references [6],[7] and [21] are compared with the proposed method as shown in Table 3. Technique of [21] processing time is the least, while the accuracy of the proposed method is close to that of [6] and [21] for good quality images, while it outperforms in case of the bad quality images. Also, Figure 15 shows examples of fingerprints from the three databases segmented by the three compared segmentation methods. The second method of [7] performs the worst.

Table 3: Comparison between segmentation methods on FOE benchmark

\begin{tabular}{|l|l|l|l|l|}
\hline \multirow{2}{*}{ Method } & \multicolumn{2}{|c|}{ Good images } & \multicolumn{2}{c|}{ Bad images } \\
\cline { 2 - 5 } & $\begin{array}{l}\text { Average } \\
\text { time }\end{array}$ & $\begin{array}{l}\text { Average } \\
\text { error }\end{array}$ & $\begin{array}{l}\text { Average } \\
\text { time }\end{array}$ & $\begin{array}{l}\text { Average } \\
\text { error }\end{array}$ \\
\hline Fen [6] & $271.5 \mathrm{~ms}$ & $4.34 \%$ & $257.8 \mathrm{~ms}$ & $15.39 \%$ \\
\hline Zhou [7] & $272.5 \mathrm{~ms}$ & $37.36 \%$ & $245.9 \mathrm{~ms}$ & $48.28 \%$ \\
\hline Peralta [ 21] & $\mathbf{2 1 0 . 3} \mathbf{~ m s}$ & $\mathbf{4 . 1 \%}$ & $\mathbf{1 9 5 . 3} \mathbf{~ m s}$ & $20.73 \%$ \\
\hline $\begin{array}{l}\text { Proposed } \\
\text { method }\end{array}$ & $250.1 \mathrm{~ms}$ & $5.03 \%$ & $235.7 \mathrm{~ms}$ & $\mathbf{1 2 . 6 2 \%}$ \\
\hline
\end{tabular}

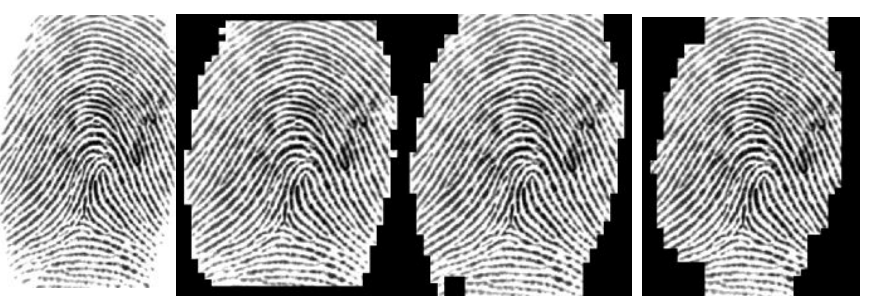

(a) (b) (d)
Figure 15: Segmentation of fingerprints (a) original fingerprint image, segmentation using (b) Fen ${ }^{(6)}$, (c) Zhou ${ }^{(7)}$, and (d) the proposed method

\section{Conclusion}

A new segmentation technique based on intensity mean and variance thresholding and morphological operation with different structuring element size is presented and proved to be more robust, and faster than the variance thresholding based techniques.

\section{References}

(1) A.M. Bazen and S.H. Gerez, "Systematic Methods for the Computation of the Directional Fields and Singular Points of Fingerprints," IEEE Trans. on Pattern Analysis Machine Intelligence, vol. 24, no. 7, pp. 905-919, 2002.

(2) D. Maltoni, D. Maio, A. K. Jain and S. Prabhakar, "Handbook of Fingerprint Recognition", second edition, chapter 3, Springer, 2009. 
(3) L. Hong, Y. Wan, and A. K. Jain, "Fingerprint image enhancement: algorithm and performance evaluation," IEEE Trans. on Pattern Analysis and Machine Intelligence, vol. 20, no. 8, pp. 777-789, 1998.

(4) Mehtre B.M., Murthy N.N., Kapoor S. and Chatterjee B., "Segmentation of fingerprint images using the directional image," Pattern Recognition, vol. 20, no. 4, pp. 429-435, 1987.

(5) A.M. Bazen and S.H. Gerez, Directional Field Computation for Fingerprints Based on the Principal Component Analysis of Local Gradients, Proc. ProRISC2000, 11th Ann. Workshop Circuits, Systems and Signal Processing, Nov. 2000.

(6) Wang Feng, Wang Xiuyou and Xu Lin, "An improved fingerprint segmentation algorithm based on mean and variance", Int. Workshop on Intelligent Systems and Applications ISA, pp.: 1-4, May 2009.

(7) J. Zhou, F. Chen, and J. Gu, "A Novel Algorithm for Detecting Singular Points from Fingerprint Images", IEEE Trans. on Pattern Analysis and Machine Intelligence, vol. 31, no. 7, pp.:1239-1250, July 2009.

(8) Bazen A.M. and Gerez S.H., "Segmentation of Fingerprint Images," in Proc. Workshop on Circuits Systems and Signal Processing (ProRISC 2001), 2001.

(9) Xinjian Chen, Jie Tian, Jiangang Cheng and Xin Yang,"Segmentation of Fingerprint Images Using Linear Classifier", EURASIP Journal on Applied Signal Processing, Hindawi Publishing Corporation, vol. 4, pp.:480-494,2004.

(10) Yang G., Zhou G.T, Yin Y., and Yang X., "K-Means Based Fingerprint Segmentation with Sensor Interoperability", EURASIP Journal on Advances in Signal Processing, vol. 2010, No. 54, pp.:1-12, February 2010

(11) Ratha N.K., Chen S.Y. and Jain A.K., "Adaptive flow orientation-based feature extraction in fingerprint images," Pattern Recognition, vol. 28, no. 11, pp. 1657-1672, 1995.

(12) C.H. Park, J.J. Lee, M.J.T. Smith and K.H. Park , "Singular Point Detection by Shape Analysis of Directional Fields in Fingerprints," Pattern Recognition, vol. 39, no. 5, pp. 839855, 2006.

(13) Fan L., Wang S., Wang H. and Guo T., "Singular points detection based on zero-pole model in fingerprint images," IEEE Transactions on Pattern Analysis Machine Intelligence, vol. 30, no. 6, pp. 929-940, 2008.

(14) Mehtre B.M. and Chatterjee B., "Segmentation of fingerprint images -A composite method," Pattern Recognition, vol. 22, no. 4 , pp. $381-385,1989$.

(15) Maio D. and Maltoni D., "Direct gray-scale minutiae detection in fingerprints," IEEE Transactions on Pattern Analysis Machine Intelligence, vol. 19, no. 1, 1997.

(16) Alonso-Fernandez F., Fierrez-Aguilar J.and Ortega-Garcia J., "An Enhanced Gabor Filter-Based Segmentation Algorithm for Fingerprint Recognition Systems," in Proc. Int. Symp. on Image and Signal Processing and Analysis, 2005.

(17) Pais Barreto Marques A.C. and Gay Thome A.C., "A Neural Network Fingerprint Segmentation Method," in Proc. Int. Conf. on Hybrid Intelligent Systems, 2005.

(18) Chikkerur S., Cartwright A.N. and Govindaraju V.,"Fingerprint enhancement using STFT analysis," Pattern Recognition, vol. 40, no. 1, pp. 198-211, 2007.

(19) Saparudin and Ghazali Sulong,"Segmentation of Fingerprint Image Based on Gradient Magnitude and Coherence", International Journal of Electrical and Computer Engineering (IJECE), Vol. 5, No. 5, October 2015, pp. 1202 1215.
(20) Pedro M. Ferreira, Ana F. Sequeira, and Ana Rebelo,"A Fuzzy C-Means Algorithm for Fingerprint Segmentation", R. Paredes et al. (Eds.): IbPRIA 2015, LNCS 9117, Springer International Publishing Switzerland 2015 pp. 245-252, 2015. DOI: 10.1007/978-3-319-19390-8 28.

(21) Daniel Peralta, Mikel Galar, Isaac Triguero, Oscar MiguelHurtado, Jose M. Benitez, Francisco Herrera, "Minutiae filtering to improve both efficacy and efficiency of fingerprint matching algorithms", Engineering Applications of Artificial Intelligence, vol. 32, pp.: 37-53, 2014.

(22) http://www.sourceafis.org/blog/nist-mindtct-and-bozorth3review/, last accessed 17/11/2015.

(23) Atul S. Chaudhari, Dr. Girish K. Patnaik, Sandip S. Patil,'Implementation of Minutiae Based Fingerprint Identification System using Crossing Number Concept", International Journal of Computer Trends and Technology (IJCTT) - volume 8 number 4- Feb 2014.

(24) Runlin Zhao,"Authentication System Based on Fingerprint Characteristics", Sensors \& Transducers, Vol. 178, Issue 9, September 2014, pp. 146-153.

(25) Kryszczuk K. and Drygajlo A., "Singular Point Detection in Fingerprints Using Quadrant Change Information," in Proc. Int. Conf. on Pattern Recognition (18th), vol. 4, pp. 594-597, 2006.

(26) G. Cao, Z. Mao, Q.S. Sun, "Core-Point Detection Based on Edge Maps in Fingerprint Images", Journal of Electronic Imaging, vol.18, no. 1, 2009.

(27) Choi H., Choi K. and Kim J., "Fingerprint Matching Incorporating Ridge Features With Minutiae", IEEE Trans. On Information Forensics and Security, vol. 6, no. 2, pp. 338345, June, 2011.

(28) N.K. Ratha, A.K. Jain and D.T. Rover, "Fingerprint Matching on Splash 2". Technical Report, Department of Computer Sience, Michigan University, East Lansing, 1994.

(29) Rafael C. Gonzalez and Richard E. Woods, Digital Image Processing, second edition, Prentice Hall, 2002.

(30) http://bias.csr.unibo.it/fvc2002/.

(31) Gongping Yang, Ying Li, Yilong Yin and Ya-Shuo Li," TwoLevel Evaluation on Sensor Interoperability of Features in Fingerprint Image Segmentation", Sensors, vol. 12, no. 3, pp.: 3186-3199, 2012.

(32) http://www.nist.gov/srd/nistsd4.cfm.

(33) http://bias.csr.unibo.it/fvc2004/ 